\title{
Indian Traffic Signboard Recognition and Driver Alert System Using Machine Learning
}

\author{
Shubham Yadav*, Anuj Patwa, Saiprasad Rane, Chhaya Narvekar \\ Xavier Institute of Engineering, Mahim Causeway, Mahim (West), Mumbai, \\ Maharashtra 400016, India \\ *Corresponding Author: shubham.yadav.5497@gmail.com
}

(Received 20-04-2019; Revised 14-05-2019; Accepted 14-05-2019)

\begin{abstract}
Sign board recognition and driver alert system which has a number of important application areas that include advance driver assistance systems, road surveying and autonomous vehicles. This system uses image processing technique to isolate relevant data which is captured from the real time streaming video. The proposed method is broadly divided in five part data collection, data processing, data classification, training and testing. System uses variety of image processing techniques to enhance the image quality and to remove non-informational pixel, and detecting edges. Feature extracter are used to find the features of image. Machine learning algorithm Support Vector Machine (SVM) is used to classify the images based on their features. If features of sign that are captured from the video matches with the trained traffic signs then it will generate the voice signal to alert the driver. In India there are different traffic sign board and they are classified into three categories: Regulatory sign, Cautionary sign, informational sign. These Indian signs have four different shapes and eight different colors. The proposed system is trained for ten different types of sign. In each category more than a thousand sample images are used to train the network.
\end{abstract}




\section{International Journal of Applied Sciences and Smart Technologies}

Volume 1, Issue 1, pages 1-10

ISSN 2655-8564

Keywords: image processing, signboard detection, svm algorithm, raspberry pi

\section{Introduction}

Recognition of signboard correctly at the right time and at the right place is very important for drivers to insure themselves and their passengers' safe journey. However, sometimes, due to the change of weather conditions or viewing angles, signs are difficult to be seen until it is too late. Now a days increases in computing power have brought computer vision to applications. On the other hand, the increase in traffic accidents accompanying the increasing amount of traffic has become a serious problem for society. The road accidents is particularly high under special road conditions, such as at the entrance to a one-way street, sharp curves, and intersections. One possible countermeasure is to install "STOP", "NO LEFT TURN" and other signs in order to notify the driver of the road conditions and other traffic information. Anyhow, there remains the possibility that the driver who is depending on his/her state of mind, fail to notice the sign while driving, a serious accident is possible if the driver fails to notice a sign such as "DO NOT ENTER", "STOP" etc [1].

It is possible that accidents can be prevented by utilizing an automatic sign board recognition system to provide traffic information to the driver, including information about the road in front of the vehicle. Signs also have distinct shapes like circles, triangles, rectangles and octagons. These systems assist drivers to drive safely. While driving the vehicle the driver gets the alert message like go slow, ahead is speed breaker. There are many detection techniques developed in recent days for traffic light and sign board detection. A system which involves detection process of traffic sign and sending the alert message does not exist. So keeping attention towards different traffic signs are difficult task for every drivers. So we proposed a system that can be used to detect traffic sign board. Traffic signs detection is an important part of driver assistant systems. These can be designed in different colors or shapes, in high contrast background. So in order to capture these images, traffic signs are oriented upright and facing camera. Hence there will be geometric and rotational distortions. In these cases accuracy is a key consideration. Any miss classified or undetected sign and lights will 


\section{International Journal of Applied Sciences and Smart Technologies}

Volume 1, Issue 1, pages 1-10

ISSN 2655-8564

produce adverse impacts on system. The basic idea of proposed system is to provide alertness to the driver about the presence of traffic sign at a particular distance apart. This system will be able to detect, recognize and infer the road traffic signs would be a prodigious help to the driver. The objective of an automatic road signs recognition system is to detect and classify one or more road signs from within live color images captured by a camera. The color of a traffic sign is easily distinguishable from the colors of the environment.

In this we provide alertness to the driver about the presence of signboard at a particular distance apart. The system provides the driver with real time information from road signs, which consist the most important and challenging tasks. Next generate an voice warning to the driver in advance of any danger. This warning then allows the driver to take appropriate corrective decisions in order to mitigate or completely avoid the event. First, it is necessary to select the hardware equipment to solve this problem. The second stage is based on color processing or object detection method based on rapid color changes. Image processing technology is mostly used for the identification of the signboards. The alertness to the driver is given as audio output.

\section{Review of Literature}

There are many algorithms and methodologies have been proposed for road traffic sign detection [2-6]. Reza Azad proposed the system with Iranian Traffic signs with detection and recognition and the letters are segmented with SVM classifier. Another method has also been proposed by Gauri Tagunde based on color and shape Features by Detection and Recognition approaches have been proposed to deal with sign board detection and recognition. Most of these systems typically involve two tasks finding the locations and sizes of sign board in natural scene images (sign board detection) and recognizing the detected signs board to interpret its meaning (sign board recognition). Being designed with regular shapes and conspicuous colours, sign board attract human driver attention so as to be easily captured by human drivers. Mohammad Amen proposes the system with YCbCr colour space and shape based filtering the detected traffic signs are tracked and recognized using interest point descriptors. The algorithm is robust and can detect signs even when the traffic sign board is rotated. The traffic sign 


\section{International Journal of Applied Sciences and Smart Technologies}

Volume 1, Issue 1, pages 1-10

ISSN 2655-8564

template database can be updated easily. The method is aimed at achieving high accuracy in recognizing traffic signs at real-time, with a low computational cost. Reduced computational complexity of the algorithm enables the implementation of the proposed method in embedded systems for driver assistance. In the case of traffic sign detection majority of system make use of colour information as a method for segmenting images. The performance of colour based road sign detection is often reduced in scenes with strong illumination, poor lightning or adverse weather conditions. The vast majority of the existing systems consist of hand label real images which are repetitive time consuming and error prone process. Information about traffic symbols, such as shape and colour, can be used to place traffic symbols into specific groups; however there are several factors that can hinder effective detection and recognition of traffic signs. These factors include variations in illumination occlusion of signs, motion blur, and weather -worn deterioration of signs. Road scene is also generally much cluttered and contains many strong geometric shapes that could easily be misclassified as road signs. Accuracy is a key consideration because even one misclassified or detected sign could have an adverse impact on the driver.

\section{Design}

Many times we see that many road accidents take place. This can be due to driver's ignorance of traffic sign board and road signs. As the road traffic is increasing day by day there is a necessity of following the traffic rules with proper discipline. Traffic signboard detection is an important part of driver assistant systems. The basic idea of proposed system is to provide real time voice signal to the driver about the presence of traffic sign board at a particular distance apart. The project is divided in to two part:

1. Training

2. Implementation

The system provides the driver with real time information from road sign board, which consist the most important and challenging tasks. It generates an voice signal to the driver in advance of any danger. This warning allows the driver to take some appropriate actions in order to avoid the accident. 


\section{International Journal of Applied Sciences and Smart Technologies}

Volume 1, Issue 1, pages 1-10

ISSN 2655-8564

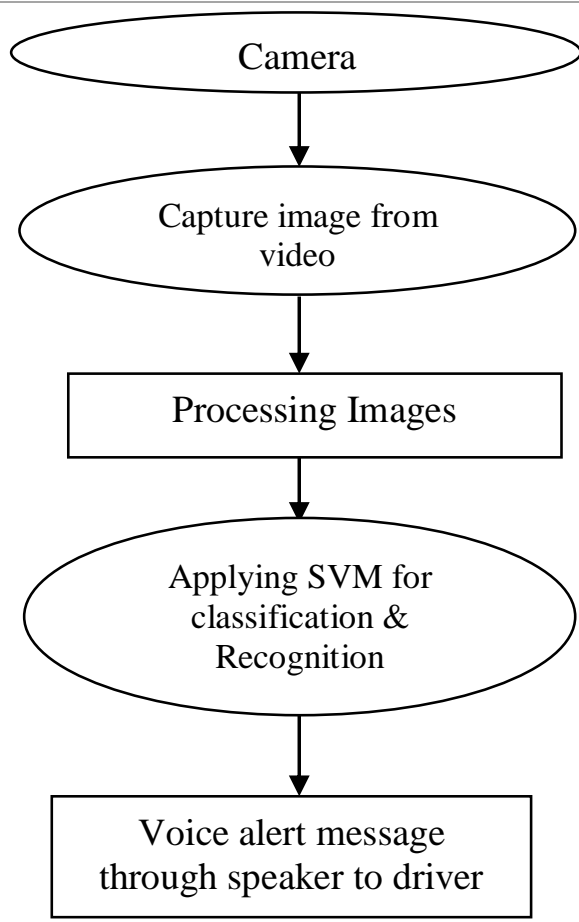

Figure 1. Flow diagram of the Proposed System

The alertness to the driver is given as a voice signal through speaker as an output. There are two method to classify the images in machine learning, convolution neural network (CNN) and Support Vector Machine (SVM). The proposed system uses support vector machine (SVM) for classification.

\section{Working of the System}

Support Vector Machine is a supervised machine learning algorithm which is also known as the linear classifier mostly used for the classification purpose. The main advantage of SVM algorithm is, its strong ability to Classify any data. When the dataset have a clear classification boundary in that situation SVM is best option than other available method.

SVM is considered as one of the best classifier and it is simple to use and understand than other classifier. The proposed system uses $90 \%$ of sample data for training and $10 \%$ for testing. The working of the system is broadly divided in three phase:

1. Color Segmentation

2. Shape Classification

3. Recognition 


\section{International Journal of Applied Sciences and Smart Technologies}

Volume 1, Issue 1, pages 1-10

ISSN 2655-8564

\section{Phase 1: Color Segmentation}

In this phase candidate blob are extracted from the input image. Color segmentation phase is important phase because color every traffic sign are such that they appears different from the surrounding environment. HSI color space of image processing techniques used for segmenting the color. This is basically detection where region of interest is identified by using image processing techniques. Using the image processing technique system creates contours on each video frame and finds ellipses and circle among those contours. Detection strategy includes, increasing the contrast of video frame, removing unnecessary colors like green with HSV color range, using Laplacian of Gaussian to display the boarder of the candidate blob, making contour by binarization and detecting the ellips like and circle like contours.

\section{Phase 2: Shape Classification}

The candidate blob that are extracted from the video frame of the segmentation phase are now need to classify. The classification of these candidate are based on the shape. For classification of the candidate blob based on the shape linear SVM is used. There are two major task involved in shape classification.

\section{Shape Feature Extraction :}

First step in shape classification is to make feature vectors for the input to the linear

SVM. Many methods have been proposed for obtaining the feature vectors (see [7, 8]). In this work, we have used distance to border vector (DtB) [8]. DtB stands for the distance of the blob from the external edge of the blob to its bounding box.

\section{Training and Testing Using Linear SVM :}

Once the feature Vector for the ROI is created then the classification is initiated. For Classification of the shape eight linear SVM is used. SVM is machine learning algorithm which can classify the data in different group. It is based on concept of decision plane where the training data is mapped to higher dimensional space and separated by plane defining two or more classes of data. The extensive introduction can befound in $[9,10]$. 


\section{International Journal of Applied Sciences and Smart Technologies}

Volume 1, Issue 1, pages 1-10

ISSN 2655-8564

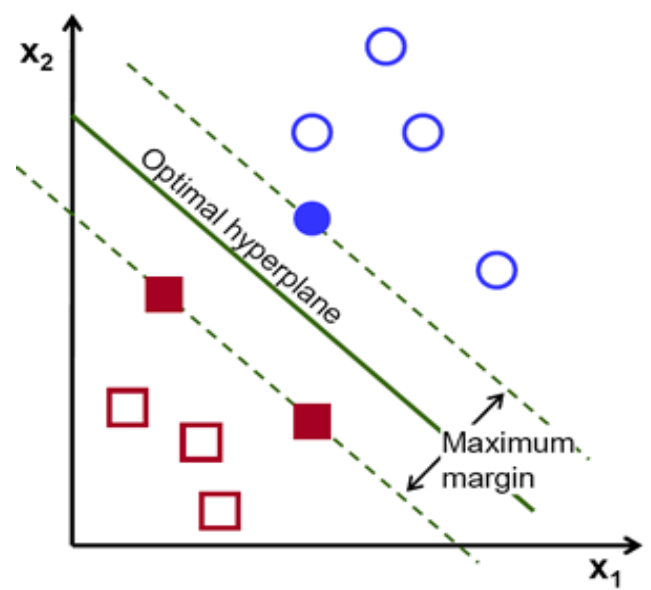

Figure 2. Shows possible hyper planes [11]

The proposed system is trained for ten traffic signs and the image of signs we can see in fg.3. In this $90 \%$ of the sample data is used to trained the system and $10 \%$ of the sample data is used for testing the system.

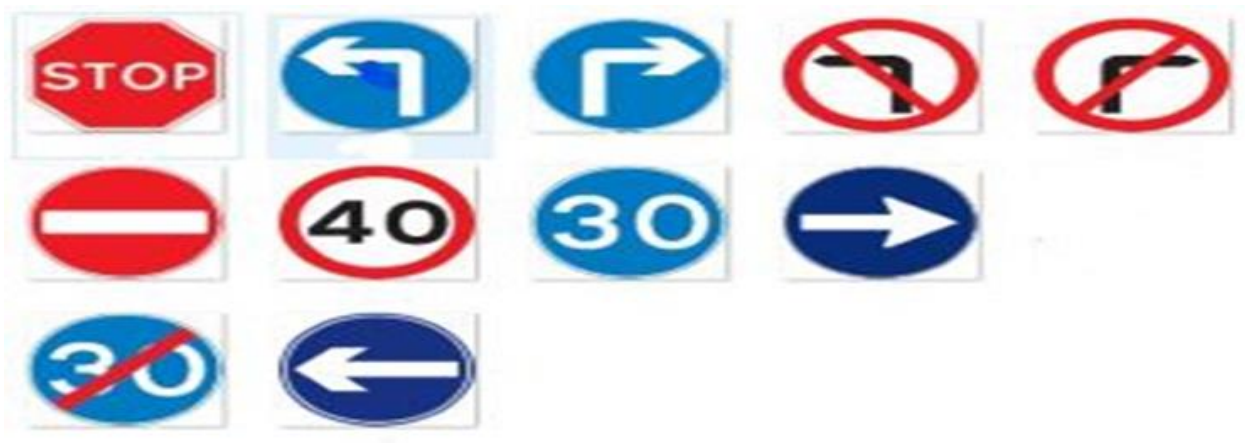

Figure 3. Trained traffic signs

\section{Phase 3: Recognition}

Once the shape classification process is done then the next step is sending the blob to pattern recognition stage. To perform the recognition of pattern the radial basis function $(\mathrm{RBF})$ is used. In this phase non linear SVM is used to recognized. In this classified blob is first converted into gray scale image then applying feature extracter to extract the features of the blob. Non linear SVM is used to recognition purpose in which extracted features are compared with all blob that are having the same shape and color. If the blob features matches with the trained signs features then system generates the 


\section{International Journal of Applied Sciences and Smart Technologies}

Volume 1, Issue 1, pages 1-10

ISSN 2655-8564

alert message calling the label of that class. The alert message is given in the form voice signal through speaker.

\section{Working Result of Proposed System}

The proposed system recognized almost all the traffic sign correctly when the traffic sign are stable while accuracy of the system is decreases while in motion. The environment and light also have the adverse effect on the system. sometimes the images which is captured from the real time streaming video have high contrast or low contrast in such cases system were not able to detect the traffic sign. So performance of the propose system in different environment not well but if the system have sample images with that environment then it works well. So we can say that accuracy of the system depend on number of sample images for a particular sign in that environment. Due to text to speech converter API some time the voice signal are delayed by few second.

\section{Conclusions}

The performance of the proposed system is quite good when system move slowly keeping signboard stationary but performance of the system while moved fast are not as per expectation. Environment and light also affect the system performance. Sometime due to text to speech converter API alert signal was getting delay. According to statistical report 3 death happens every 10 minutes due to road accident in India. On successful implementation of this project we expect to drastic reduction in road accident

\section{References}

[1] G. Revathi and G. Balakrishnan, "Indian sign board recognition using image processing techniques," International Journal of Advanced Research in Biology Engineering Science and Technology, 2 (15), 326-330, 2016.

[2] R. Azad, B. Azad, and I. T. Kazerooni, "Optimized method for Iranian road signs detection and recognition system," International Journal of Research in Computer Science, 4 (1), 19-26, 2014.

[3] K. M. Sumi and K. M. N. Arun, "Detection and recognition of road signs," International Journal of Computer Applications, 160 (3), 1-5, 2017. 


\section{International Journal of Applied Sciences and Smart Technologies}

Volume 1, Issue 1, pages 1-10

ISSN 2655-8564

[4] A. P. T. Agnes, C. A. Aiswarya, A. Augustine, A. S. Kumar, and N. Aswathy, "Real time traffic light and sign board detection," International Journal of Engineering Research and General Science, 5 (3), 50-57, 2017.

[5] W. Zhang, "Shift-invariant pattern recognition neural network and its optical architecture," Proceedings of Annual Conference of the Japan Society of Applied Physics, p. 734, 1988.

[6] https://medium.com/@RaghavPrabhu/understanding-of-convolutional-neuralnetwork-cnn-deep-learning-99760835f148 (Accessed on 14-05-2019).

[7] P. G. Jiménez, H. G. Moreno, P. Siegmann, S. L. Arroyo, and S. M. Bascón, "Traffic sign shape classification based on support vector machines and the FFT of the signature of blobs," Proceedings of the 2007 IEEE Intelligent Vehicles Symposium, 375-380, Istanbul, 13-15 June 2007.

[8] S. L. Arroyo, P. G. Jiménez, R. M. Bascón, F. L. Ferreras, and S. M. Bascón, “Traffic Sign Shape Classification Evaluation I: SVM using Distance to Borders," Proceedings of IEEE Intelligent Vehicles Symposium, 557-562, Las Vegas, June 2005.

[9] S. Abe, Support Vector Machines for Pattern Classification, Springer, London, 2005.

[10] C. C. Chang and C. J. Lin, LIBSVM: A Library for Support Vector Machines, 2001, https://www.csie.ntu.edu.tw/ cjlin/papers/libsvm.pdf (Accessed on 14-05-2019).

[11] https://towardsdatascience.com/support-vector-machine-introduction-to-machinelearning-algorithms-934a444fca47 (Accessed on 14-05-2019). 
International Journal of Applied Sciences and Smart Technologies

Volume 1, Issue 1, pages 1-10

ISSN 2655-8564

This page intentionally left blank 